\title{
Empirical Study of Research Issues and Challenges in Cloud Computing for E-Health Systems- A Review
}

\author{
${ }^{1}$ Shikha Mittal, ${ }^{2}$ Dr. Paramjeet Singh, ${ }^{3}$ Dr. Rahul Malhotra \\ ${ }^{I}$ Ph.D Scholar, Dept. of CSE, IKGPTU, Kapurthala \\ ${ }^{2}$ Associate Professor, Dept. of CSE, GZSCCET, Bathinda \\ ${ }^{3}$ Director-Principal, SDDIET, Barwala
}

\begin{abstract}
Cloud Computing could be a hot research area among the researchers in today's world. It is an internet-based pool of heterogeneous resources. Cloud is much more reliable to make availability of resources when required for online users. Resources are provided as a service on demand with pay-on-usage basis. Computing is done on clouds for less cost and maximum utilization of resources. The cloud computing during this paper has been explored the variety of mechanism for providing data security in order that it would be widely accepted by the variety of many users. The aim of the paper is to present cloud computing service models, service providers, deployment models along with research issues and challenges in cloud computing for e-health systems.
\end{abstract}

Keywords- Cloud Computing, Service Models, Deployment Models, E-Health Cloud

\section{INTRODUCTION}

In the recent years, for storing the huge amount of data through World Wide Web (WWW) the only most famous application is Cloud computing. Because of the ability of Cloud computing, the users are increasing vastly in the recent time. Cloud computing also provides the better effective application with the adequate cost in a satisfied way for the users. It is a web-based service and a strategy for giving ondemand services, expandable and perfectly elastic software services using the internet. Cloud computing is a scalable and unambiguous like user could increase and decrease the resources at anytime anywhere. It could be a consistent access to nearly limitless resources and makes a good vary of solutions obtainable to us. A cloud could be a style of parallel and distributed system. It comprises of the gathering of interconnected systems. It is virtualized computers that are dynamically provided together or additional computing resources. Cloud computing could be a new manner of considering IT services and how resources could be utilized in a broader way. Solutions and services that are bought and consumed in real time over the online are considered as cloud services. Cloud computing would be a distributed vogue that centralizes server resources on a climbable platform therefore on offer on-demand computing resources and services because of the unprecedented success of internet in a very previous few years, computing resources square measure presently lots of ubiquitously accessible. And it enabled the conclusion of a replacement computing thought called Cloud computing. An exceptional characteristic of cloud computing is it pay per use one as the cloud user is only required to pay just for the used services [20]. Cloud Computing has been attracting the enormous companies such as- Google, Microsoft and Amazon and thought of as a good influence in today's data science technology world. The Cloud computing environment is shown is fig. 1 below.

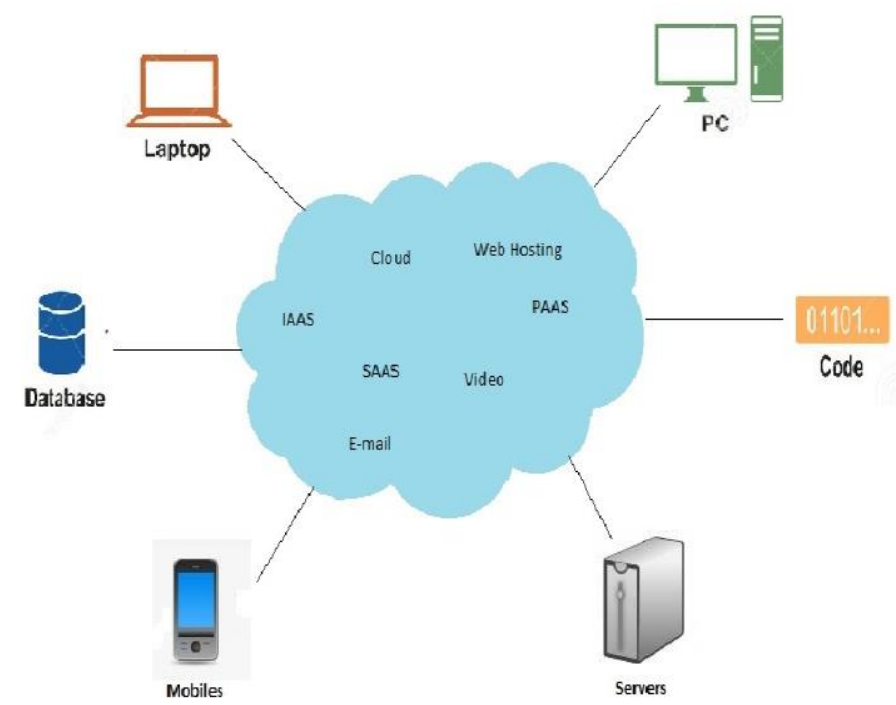

Fig.1 Cloud Computing Environment

The remainder of the paper is organized into different sections as follows: Section-II presents a brief background of cloud computing service models and their service providers. Section-III explains the deployment models of cloud computing along with 


\section{International Journal of Research in Advent Technology, Vol.7, No.5, May 2019 E-ISSN: 2321-9637 \\ Available online at www.ijrat.org}

necessary parameters of Private and Public cloud. Section-IV discusses a brief summary of literature review. Section-V covers the research methodology used in the paper. Section-VI presents research issues and challenges. Conclusion is shown in Section-VII while references are given at the last.

\section{CLOUD SERVICE MODELS}

Cloud computing service models are generally categorized into three major categories such as- PaaS, IaaS, and SaaS. These are also called as service model of Cloud computing [2]. Cloud provides models for using these services for the users [1]. Fig. 2 depicts the Cloud computing service models.

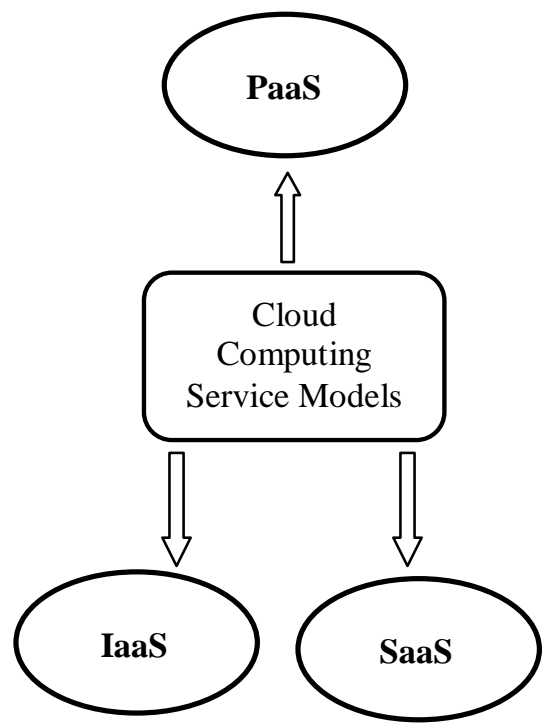

Fig. 2 Cloud Computing Service Models

Now we discuss these models in detail one by one.

\section{A. PaaS (Platform As A Service)}

Gartner defines PaaS as a cloud service model wherever the cloud is employed to deliver a platform to users from that they will develop, initialize and manage applications. PaaS offerings generally embrace a base software package and a collection of applications and development tools. PaaS eliminates the necessity for organizations to create and maintain the infrastructure traditionally used to develop applications. Paas is typically known as 'middleware', pertaining to however it conceptually sits somewhere between SaaS and IaaS. PaaS providers offer a predefined combination of OS and application servers, such as LAMP platform (Linux, Apache, MySql and PHP), classified J2EE, Ruby etc. Google's App Engine, Force.com etc. In PaaS no need of separate testing and developing environment. Some PaaS like Microsoft Azure and Google Appengine match the user applications on-demand [21]. Examples of PaaS are- Google App-Engine,
Force.com, and Azure from Microsoft. The PaaS service model is shown in fig.3.

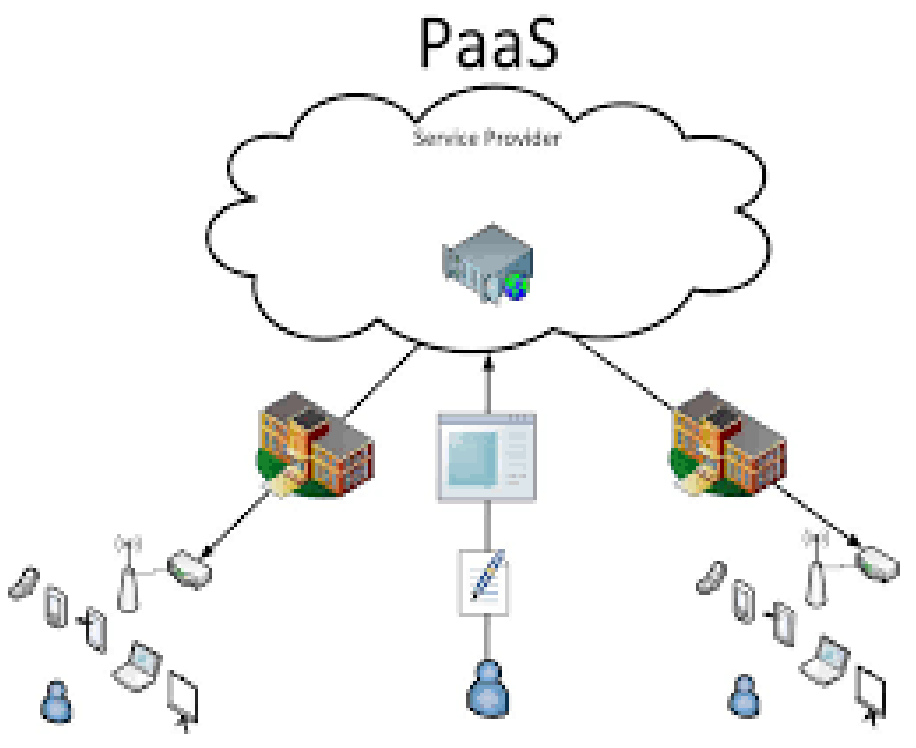

Fig.3 PaaS Service Model

Some of the [6] PaaS service providers are as under:

- Google App Engine by Google Cloud services from Google.

- Windows Azure PaaS services by Windows Azure from Microsoft.

- Amazon Elastic BeanStalk by Amazon Web Services from Amazon.

- Openshift by Red Hat from Linux.

- Engine Yard run on Amazon Web Services by Amazon.

\section{B. IaaS (Infrastructure As A Service)}

IaaS is the foundation for every computing infrastructure. It is a service provider to the clients [22]. IaaS is that the lowest-level of cloud service process and arguably the most necessary. With IaaS, pre-configured hardware resources are provided to users through a virtual interface, not like PaaS and SaaS, IaaS doesn't embody applications or perhaps an operating system (implementing all of that's left up to the customer), it merely gives access to the infrastructure required to power or support that computer code. IaaS will offer additional storage for company knowledge backups, network bandwidth for an organization website server, or it will even alter access to high power computing that was antecedently solely accessible to those with supercomputers. Examples of IaaS are- Amazon EC2, Go grid. The IaaS service model is shown in fig.4. 


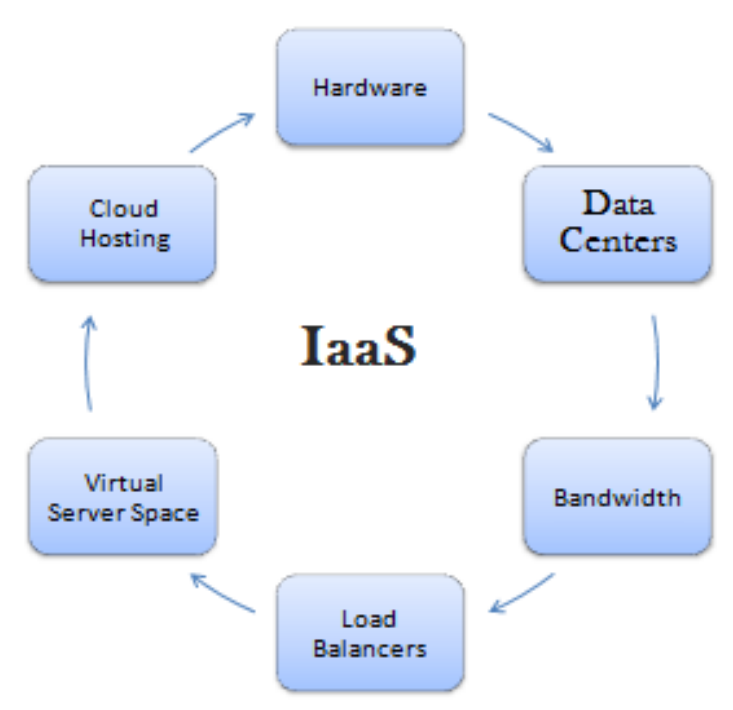

Fig.4 IaaS Service Model

Some of the [6] IaaS service providers are as under:

- Amazon Elastic Cloud Compute (EC2) service from Amazon Web Services by Amazon.

- Google Compute Engine from Google Cloud Services by Google.

- Windows Azure Virtual Machines from Windows Azure by Microsoft.

- IBM Smart Cloud Enterprise by IBM.

- HP Enterprise Converged Infrastructure from HP.

\section{SaaS (Software As A Service)}

Sometimes remarked as 'on-demand software', SaaS may be a software licensing and delivery model wherever a totally purposeful and complete product is delivered to users over the net on a subscription basis. SaaS offerings are generally accessed by finish users through an online browser (making the user's operating system for the most part irrelevant) and maybe beaked supported consumption or, a lot of merely, with a flat monthly charge. SaaS offerings are the foremost wide visible of all the cloud computing service models. In fact, several users may be using SaaS products while not even realizing. With SaaS, a provider sells an application to customers on license basis, in a "pay-as-you-go" model [3]. Examples of SaaS are- Salesforce.com, Google Mail, and Google Docs $[4,5]$. The SaaS service model is shown in fig.5.

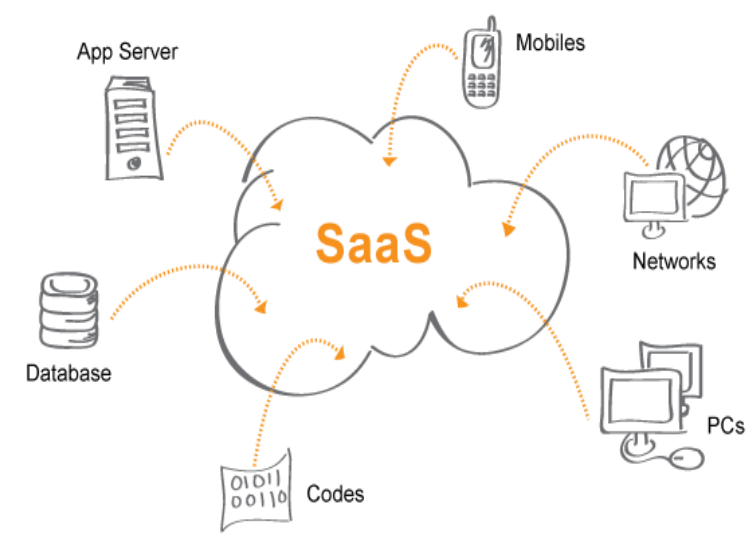

Fig.5 SaaS Service Model

Some of the [6] SaaS service providers are as under:

- Salesforce CRM from Salesforce.

- Oracle CRM from Oracle On-Demand from Oracle.

- SAP ERP and SAP CRM by SAP Business By Design from SAP.

- SaaS applications and services from Cloud9 Analytics.

\section{CLOUD DEPLOYMENT MODELS}

The model for the deployment in the cloud computing is list out as four models. They are as follows:

- Private

- Public

- Hybrid

- Community

\section{- $\quad$ Private Cloud}

If the single organization is owned a cloud infrastructure which is operated only within this organization is called the private cloud infrastructure. For example Microsoft, HP and the Intel are having their own private clouds.

\section{- Public Cloud}

If the cloud service is marketing to the public otherwise to the large scale industry, then it is termed as the public cloud infrastructure. For example Microsoft Azure and the Amazon Web Services are the public clouds.

\section{- Hybrid Cloud}

If the two or more different types of clouds (private, public, community) are encountered by a consistent or exclusive method to improve the portability of the information, this is termed as the hybrid cloud infrastructure.

\section{- Community Cloud}

If the cloud service is shared by the different organizations which has the common mission, vision, security requirements, terms and policies, agreement conditions and the goals for provide the support to a particular community are usually termed as the 


\section{International Journal of Research in Advent Technology, Vol.7, No.5, May 2019 E-ISSN: 2321-9637 \\ Available online at www.ijrat.org}

community cloud infrastructure. For example: Google Gov.

\subsection{Private vs. Public Cloud}

\begin{tabular}{|c|l|l|l|}
\hline \multicolumn{4}{|c|}{ Table -1: Private vs. Public Cloud } \\
$\begin{array}{c}\text { Sr. } \\
\text { No. }\end{array}$ & Attributes & Private Cloud & Public Cloud \\
\hline 1. & Infrastructure & $\begin{array}{l}\text { Available for public and many } \\
\text { group of organization }\end{array}$ & $\begin{array}{l}\text { Deployed and operated only for } \\
\text { an organization }\end{array}$ \\
\hline 2. & Resources & Privately shared & Publicly shared \\
\hline 3. & Security & $\begin{array}{l}\text { Suitable for high confidential } \\
\text { data }\end{array}$ & $\begin{array}{l}\text { Suitable for less confidential } \\
\text { data }\end{array}$ \\
\hline 4. & Cost & High-cost & Low-cost \\
\hline 5. & Tenants & Solo tenant & Multiple tenants \\
\hline 6. & Computing model & Grid computing & Utility computing \\
\hline 7. & Scalability & $\begin{array}{l}\text { Sacrifices scalability but } \\
\text { provides greater control and } \\
\text { security }\end{array}$ & Instant and unlimited \\
\hline 8. & Performance & \begin{tabular}{l} 
Very High \\
\hline
\end{tabular} & \\
\hline
\end{tabular}

\section{LITERATURE REVIEW}

It is practically impossible to review all the available researches and past literatures which are related to cloud computing domain highlighting security and privacy research issues in e-health systems. Consequently, we succeeded in reviewing selected articles. The literature review has been carried out in this section. A brief review of some recent research works is presented here.

In[7] found some prime security attacks and supply attainable solutions for clouds: XML signature wrapping attacks, browser security, and vendor lock in. they need been known problems and evaluated existing attainable solutions to those problems to settle on the most effective one among them mentioned in their paper. A model for utility composition in cloud computing, this version was based mostly altogether on an illustration of manipulating ideas of an application domain via the bpm approach, particularly operations and static principles wont to describe the properties of business processes to assess the performance of this idea, a sequence of experimental tests became dispensed. When compared with a not composite model (NC) because of the usual approach on this place, the results showed that $\mathrm{AC}$ surpasses $\mathrm{NC}$ in terms of response time to time with the smallest complexity [8]. A framework to unravel numerous moral and security aspects associated with cloud computing [9]. A Hadoop cluster and uses the MapReduce framework to style the encryption service system. In addition, they offer a whole encryption scheme, a selective encryption scheme, and a partial encryption scheme to inscribe the video data. As a result, not
We have discussed about the public and private cloud now Table-1 shows the some basic difference between private cloud and public. solely improves the speed of video encryption however additionally optimizes the video encryption strategy. Moreover, the user will choose the encryption scheme consistent with their necessities. The experimental results show that the video encryption service that they offer will meet the wants of high-speed, security so on [10]. Security risks associated issues in cloud computing and broadminded steps that an enterprise will like scale back security risks and defend their resources. They have also clarified cloud computing qualities/advantages, shortcomings, and appropriate regions in data chance management. In expansion, cowls the advantages and disadvantages of the methodology of cloud computing. Additionally, tackles the necessary side of security involved challenges that the researchers and authors face among the protection of cloud computing [11]. The issue faced by on-line education interaction and constructs a web education interactive platform model. By analyzing the platform functions and data processing, the paper realizes the high strength of online instructors and learner's Interactive functions [12]. Most of the research work offers together with the identification over challenges within cloud forensics and between addition, the projected choices in retentive at intervals composition rely upon Cloud Service supplier (CSP) for rhetorical inquisitors. The dependence on CSP includes the gathering of data for the forensics methodology and there's conjointly a chance for sterilization data that affects the complete investigation methodology. For mitigating the dependency on CSP, a brand new model for grouping forensic evidence outside the cloud atmosphere is developed [13]. Comprehensive review of this progressive and thus the newest developments on 


\section{International Journal of Research in Advent Technology, Vol.7, No.5, May 2019 E-ISSN: 2321-9637 \\ Available online at www.ijrat.org}

mobile cloud computing underneath the $5 \mathrm{G}$ era, that helps beginning-stage researchers to possess an outline of the present solutions, techniques, and applications and investigate open analysis problems and future challenges in this domain [14]. A cloudstyle reference model that contains an enormous variety of security controls and best practices, and a cloud security assessment Model-Cloud-Trust that estimates high-level safety metrics in accordance with quantify the degree concerning confidentiality then integrity offered by using a CCS or Cloud Service Provider (CSP). Cloud-Trust is used in imitation of assess the protection level regarding four multi-tenant IaaS cloud architectures equipped with diverse cloud safety controls. Results show the probability of CCS penetrates on is high if the smallest amount set of security controls are implemented. CCS penetration likelihood drops considerably if a cloud defense thorough security style is adopted that protects virtual machine (VM) photos at rest, strengthens CSP and cloud tenant supervisor access controls, that employs altogether totally different network security controls to scale back cloud network surveillance and discovery of live VMs [15].

IlangoSriram et al. [16] go through more definitions of cloud computing and proposed the following definition:

Clouds are a large pool of easily usable and accessible virtualized resources (such as hardware, development platforms and/or services). These resources can be dynamically reconfigured to adjust to a variable load (scale), allowing also for optimum resource utilization. Typically these pools of resources exploited by a pay-per-use model in which guarantees are offered by the Infrastructure Provider by means of customized SLAs.

Imran Ashraf [17] states that cloud computing are being embraced by many fields and is most adoptable field of information technology at the moment. Due to its pay-per-use principle it is becoming very attractive and competitive solutions for even small organizations who cannot afford to have their own hardware or software infrastructure. Moreover, it's on demand changing scalability has made it very viable solution for organizations who need to change their acquired services with the changing workload. Apart from being simple and easy to use it has other advantages over traditional framework. It works in distributed environment and serves the user according to his needs. Users just need to have internet to connect to the services and it is accessible from everywhere. It has minimized cost, improved throughput, and fast access of software and hardware resources and can scale readily and easily as required. Eugene Gorelik[18] proposed that cloud computing begin with the huge IT transformation in history, and this transformation has opened many new business opportunities. It is expected that public clouds will provide most of the opportunities for cloud service providers. According to a recent survey conducted by Morgan Stanley, the percentage of companies using a public cloud is expected to rise to $51 \%$ though 2013 .

Santosh Kumar and R.H. Goudar [19] states that cloud computing is the development of parallel computing, distributed computing, grid computing, and is the combination and evolution of virtualization, utility computing, Software-as-a-Service (SaaS), Infrastructure-as-a-Service (IaaS) and Platform-as-aService (PaaS). Cloud is a metaphor to describe web as a space where computing has been pre-installed and exist as a service, data, operating systems, applications, storage and processing power exist on the web ready to be shared. To users, cloud computing is a Pay-per-Use-On-Demand mode that can conveniently access shared IT resources through the Internet.

\section{RESEARCH METHODOLOGY}

\subsection{Problem Identification}

The method which is utilized in e-health cloud systems had some drawbacks such as scalability in key management, risk of privacy exposure, flexible access and user revocation. Also the main disadvantage is that whether the patients are controlling the sharing of sensitive personal health information specifically when they are stored on the cloud server. The security of the sensitive information is the main concern to handle it. To overcome these problems, a secure E-health record cloud model (EHR) database storage in cloud environment has been proposed in our research work.

\subsection{Motivation}

The service of the health care unit is the most essential service for the people nowadays. There is a necessary need to store the sensitive information related to the patient's medical history as in a secure way. So the research and development in the e-heath cloud systems like Personal Health records and Electronic Health records is negligible area. Hence the most robust techniques of encryption and decryption should be encountered.

\subsection{Basic Model of E-Health Cloud System}

In this section, a basic simple model of e-health cloud system is discussed. We have observed that already existing models of e-health cloud in the world wide such as- ICW LifeSensor3, Google Health1, Microsoft HealthVault2 and Google Health1. In these systems the patients are used to save the data related to their health condition on 
the particular web servers. This record usually termed as the Personal Health Record (PHR) i.e. E-Health cloud. In this type of model the patients can track, gather and also accomplish the data associated with the health on the online sites. The data comprises the particular dates and the duration of the illness, the date on which the appointments made with the doctors, and it may include any other information linked with the health. The patients also include the data in their respective Personal Health Records (PHRs) the documents which are received from the health professionals for example laboratory tests reports, X-ray reports, scan reports. Fig. 6 shows the basic model of E-health cloud.

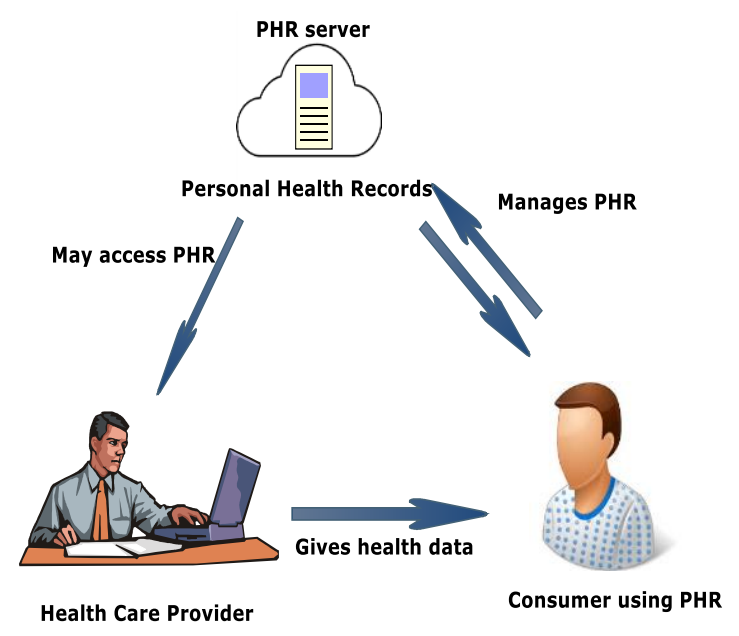

Fig.6 Basic Model of E-Health Cloud

\subsection{Objectives of Basic Model of E-Health Cloud}

Following are the key objectives of the basic model of E-health cloud-

- The E-health cloud model generally stored in a third party server in the cloud computing environment. It is responsible for the data protection belongs to the Personal Health Record (PHR) service provider.

- The patients describe the access rights based on the role for the separate health professionals. It considers if they describe the whole access to their respective doctor, the other persons like fitness trainer cannot get the whole data. They are restricted to access the some vital data.

- Due to the centralized system, the PHR is manageable from anywhere anytime. This is the main advantage of the Personal Health Record (PHR).

- The one doctor's test results can easily access with another doctor for the particular doctor if the data is already stored in the Personal Health Record (PHR).

- This way of examination reduces the time and cost of the second opinion. The person, who is managing his Personal Health Record (PHR) by his own, could know their health condition in up to date. This is used to minimize the cost of the healthcare in long term.

\section{ISSUES \& CHALLENGES IN CLOUD COMPUTING}

Although cloud computing has quickly come into the existence. The researches of cloud computing are still in an early stage. Many issues have not been resolved and new challenges have been emerging in every industry day-by-day. We discuss some of the new research issues and challenges in this section.

\subsection{Research Issues}

Some of the research issues which need critical considerations are as per the following:

- Data Breach: Confidential, sensitive and protected data is obtaining by the unwanted user or a hacker. For example, WWE wrestling was recently the victim of a security breach that leaked the private information to three million users.

- Denial of Service (DoS): Denial of service attack is to shut down the systems or network in order overpower the victim resources and make it difficult for authorized user to use.

- Internet Protocol: Many susceptibilities inside in IP such as ARP spoofing, IP spoofing, DNS Poisoning are real threats.

- Malicious Insider: A decided insider can discover more approaches to attack and cover the track in a cloud situation.

- SQL Injection Vulnerabilities: Vulnerabilities, as an example, OS injection, SQL injection and LDAP injection at the management layer will cause important problems for completely different cloud customers.

- API and Browser Vulnerabilities: Any weakness in cloud supplier's API or Interface represents a major risk once combined with social engineering or browser-based attacks the harm are often vast.

- Changes to Business Model: Cloud Computing can be a critical change to a cloud consumer's business model. IT department and business needs to adjust or confront introduction to chance. 
International Journal of Research in Advent Technology, Vol.7, No.5, May 2019 E-ISSN: 2321-9637

Available online at www.ijrat.org

\subsection{Research Challenges}

Following are a few research challenges in cloud computing:

- Cloud data management

- Security Data Encryption

- Virtual machines migration

- Access controls

- Service level agreement (SLA)

- Multi-tenancy

- Reliability and availability of services

\section{CONCLUSION}

Cloud computing is a rising technology that is growing day by day. Cloud computing provides smart measurability, service on-demand, computing capability and benefits of resources at economical scale, with flexibility, scalability and multi-tenancy. However, it additionally brings many problems like security, privacy, legal problems and lots of additional. This paper discusses various services and deployment models which are provided by the cloud computing and service providers who provide these types of services in detail. In addition, a research methodology for e-health cloud model is proposed in this paper. This paper also tackles the important aspects of research challenges and issues which the researchers and authors are generally facing in the data security of cloud computing.

\section{REFERENCES:}

[1] Zhang, Q., Cheng, L. \& Boutaba, "Cloud computing: state-of-the-art and research challenges", Journal of Internet Services and Applications (2010), Springer;

[2] S. Hameetha Begum, T. Sheeba, S.N. Nisha Rani, "Survey on cloud computing", International Journal of Advanced Research in Computer Science and Software Engineering, Vol. 3, Issue 1, pp 18-22, January 2013.

[3] Sara Qaisar, Kausar Fiaz Khawaja, "Cloud computing: network/security threats and countermeasures", Interdisciplinary Journal of Contemporary Research in Business, Vol.3, No 9 January 2011.

[4] Zhengxiong Hou, Xingshe Zhou, Jianhua Gu,Yunlan Wang, Tianhai Zhao, "ASAAS: Application Software as a Service for High Performance Cloud Computing," 12th IEEE International Conference on High Performance Computing and Communications, 2010.

[5] Wei-Tek Tsai, Xin Sun, Janaka Balasooriya, "Service-Oriented Cloud Computing Architecture," Seventh International Conference on Information Technology, 2010.

[6] Chinthagunta Mukundha, K. Vidyamadhuri , Cloud Computing Models : A Survey, Advances in Computational Sciences and Technology ISSN
0973-6107 Vol. 10, Number 5 (2017) pp. 747761

[7] Alex ME, Kishore R, "Forensics framework for cloud computing", Comput Electr Eng (2017) 60:193-205.

[8] Aljawarneh SA, Alawneh A, Jaradat R, "Cloud security engineering: Early stages of SDLC", Future Generation Computer System (2017) 74:385-392.

[9] Alshammari A, Alhaidari S, Alharbi A, Zohdy M, "Security Threats and Challenges in Cloud Computing", 2017 IEEE 4th Int Conf Cyber Secur Cloud Comput 46-51.

[10] Gonzales D, Kaplan JM, Saltzman E, et al. "Cloud-Trust-a Security Assessment Model for Infrastructure as a Service (IaaS) Clouds", IEEE Trans Cloud Comput (2017) 5:523-536.

[11] Jiugen Y, "Research on Interactive Application of Online Education Based on Cloud Computing and Large Data"(2017) 593-596

[12] Kajaree D, Behera R., "A Survey on Web Crawler Approaches", Int J Innov Res Comput Commun Eng (2017) 5:1302-1309.

[13] Malgey S, Chauhan P, "A Review on Security Issues and their Impact on Cloud Computing Environment. Int $\mathrm{J}$ Adv Res Comput Commun Eng (2016) 5:249-253.

[14]Pei D, Guo X, Zhang J A, "Video Encryption Service Based on Cloud Computing", Sadok L, Okba K, Oueslati W (2017) Management) by composition of applications. 144-150

[15] Skourletopoulos G, Mavromoustakis CX, Mastorakis G, et al., "Advances in Mobile Cloud Computing and Big Data" (2017) in the 5G Era. 22.

[16] IlangoSriram, Ali Khajeh-Hosseini, "Research Agenda in Cloud Technologies", https://arxiv.org/ftp/arxiv/papers/1001/1001.3259 .pdf.

[17] Imran Ashraf, “An overview of Service models of Cloud computing”, http://ijmcr.com/wpcontent/uploads/2014/08/Paper18779-783.pdf.

[18]Eugene Gorelik, "Cloud Computing Models", https://edisciplinas.usp.br/pluginfile.php/ 234466/mod_resource/content/2/2013-01-2.pdf.

[19] Santosh Kumar and R. H. Goudar, "Cloud Computing- Research Issues, Challenges, Architecture, Platforms and Applications: A Survey", http://www.ijfcc.org/papers/95F0048.pdf.

[20] Akande, A.O., et el., 'Management Issues with Cloud Computing', in Proceedings of the Second International Conference on Innovative Computing and Cloud Computing, 2013, ACM.

[21] A.M Chandrashekhar and K. Raghuveer, "Hard Clustering V/s. Soft Clustering: A Close Contest for Attaining Supremacy in Hybrid NIDS 
International Journal of Research in Advent Technology, Vol.7, No.5, May 2019 E-ISSN: 2321-9637

Available online at www.ijrat.org

Development", Proceedings of International Conference on Communication and Computing (ICCC-2014), Elsevier science and Technology Publication.

[22] A.M. Chandrashekhar and K. Raghuveer, "Confederation of FCM Clustering, ANN and SVM Techniques of Data mining to Implement Hybrid NIDS Using Corrected KDD Cup Dataset", Communication and Signal Processing (ICCSP) IEEE International Conference, 2014, Page 672-676. 